\title{
INFLUENCE OF HEATING TEMPERATURE ON THE ALUMINUM USED FOR ELECTRIC CABLES
}

\author{
$\underline{\text { L. Fellah }}^{1 *}$, A. Diha ${ }^{2}$ \\ Scientific Supervisor: Prof., Dr. Z. Boumerzoug ${ }^{3}$ \\ ${ }^{1}$ Department of Earth and Universe Sciences/ University of Kasdi Merbah, 30000 Ouargla, Algeria \\ ${ }^{2}$ Department of mechanic/ University sheik Larbi Tébessi/ Tébessa, 12000, Algeria \\ ${ }^{3}$ LMSM, Department of Mechanical Engineering/ University of Biskra, 07000 Biskra, Algeria \\ *E-mail : fellahcene@yahoo.fr
}

\begin{abstract}
In this study, we present the effects of temperature rise on microstructural evolution of industrial Al wire. This material is used to manufacture electric cables. For the X-ray analysis of the line profile (LPA), we have chosen the so-called «breadth method» whose basic principle is to draw the Williamson-Hall plot. We have observed that the increase in temperature causes the increasing of coherent diffraction domains (crystallites) size, interplanar distance and cell parameter. There is an expansion of the unit cell. XRD patterns refinement were performed using the whole powder pattern modelling procedure implemented in PM2k software.
\end{abstract}

Introduction. The usage on large scale of industrial aluminum for electric cables is justified by mechanical, chemical and physical characteristics of this material. ENICA-Biskra Company uses industrial aluminum in manufacture of electric cables. In this paper, thermal stress effect and its impact on industrial aluminum wires were investigated systematically by XRD techniques. Based on known material properties, lattice parameter, interplanar distance, coherent diffraction domain (crystallite) size, and strain were calculated using Line Profile Analysis (LPA) analyses. Williamson-Hall analysis method was used to study the contributions of crystallite sizes and lattice strain on the peak broadening of the material. We have performed the $\mathrm{X}$-ray diffraction patterns refinement using the recently proposed numerical fitting procedure, the whole powder pattern modelling (WPPM) implemented in PM2k software package [1].

Experimental procedures. The material investigated in this study is an industrial aluminum (99.80 wt. \%). which used in the cable factory to produce electric wires for different purposes (eg. cables). The samples studied were taken directly from the production line of the factory. The samples were cut from a wire rod of $9.5 \mathrm{~mm}$ diameter, it had a parallelepipedic shape $(10 \times 6 \times 3 \mathrm{~mm})$. For the characterization, the samples were polishedusing a mechanical polishing machine of type "MECAPOL P $220 \mathrm{~S}$ " with abrasive paper of different particle sizes (number of paper from 80 to 1200) and finished with addition of lubricant and diamond paste. The diffractograms were carried out from flat polished surfaces of samples by X'Pert Philips (Panalytical) X-ray diffractometer equipped witha furnace.

Theoretical overview. During mechanical or thermal treatment of a metal and its alloys, the microstructural parameters of the material change. Dislocations density, stacking fault probability, strain and crystallite size increase or decrease according to conditions of this treatment. X-rays can be used to assess the crystallite size and strain from the line profile of one (or more) peak(s). The particle size broadening to obey the Scherrer law [2]: $\beta_{\mathrm{S}}=\mathrm{K} \lambda / \mathrm{D} \cos \theta$, where $\lambda$ is the $\mathrm{X}$-ray wavelength and $\mathrm{D}$ is a mean linear dimension of the particle.Strain-induced broadening arising from crystal imperfections and distortion are related by equation: $\beta_{\mathrm{d}}=$ $=4 \varepsilon \tan \theta$. 
The assumption of the Williamson-Hall method is that the whole line broadening $\beta_{\mathrm{hkl}}$ of sample is a sum of the line broadening $\beta_{\mathrm{S}}$ connected to the crystallite size and the line broadening $\beta_{\mathrm{d}}$ connected to the lattice strain, according to Equation [3]: $\beta_{\mathrm{hkl}}=\beta_{\mathrm{S}}+\beta_{\mathrm{d}}$, where $\beta_{\mathrm{hkl}}$ is the total integral breadth, $\beta_{\mathrm{S}}$ and $\beta_{\mathrm{d}}$ are, respectively, the integral breadth components for size and strain. The classical Williamson-Hall plot $[3,4] \beta_{\mathrm{hkl}} \cdot \cos \theta$ versus $\sin \theta$ provides estimated values for the apparent crystallite size $<\mathrm{D}>$ and the upper limit for the lattice strain $(\varepsilon)$ from the intercept and slope, respectively: $\beta_{\mathrm{hkl}} \cdot \cos \theta=\mathrm{K} \lambda / \mathrm{D}+4 \varepsilon \sin \theta$.

Experimental results and discussion. XRD analysis. The XRD diffractogram of the studied sample before the investigation is shown in Fig. 1. We found the peaks characteristic of Al, namely the peaks (111), (200), (220), (311), (222), (400), (331) and (420), in accordance with the Aluminum ASTM sheet. The lattice parameters of the sample (as received) are summarized in Table 1. Fig. 2 shows a shift of the peaks towards the small angles and their broadening as the temperature increases. This has been interpreted by the expansion of the volume of the elementary cell and the introduction of distortions in the crystal lattice of material.

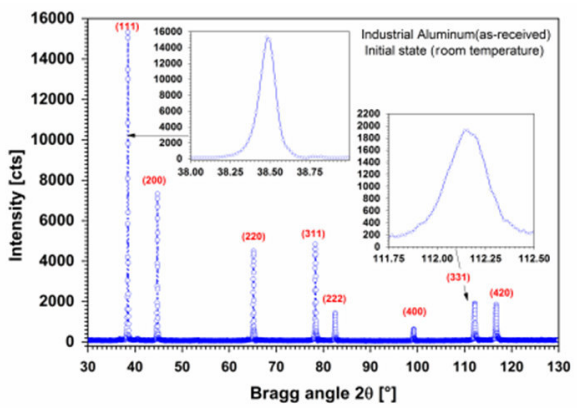

Fig. 1. X-ray pattern of industrial aluminum (as received) at room temperature

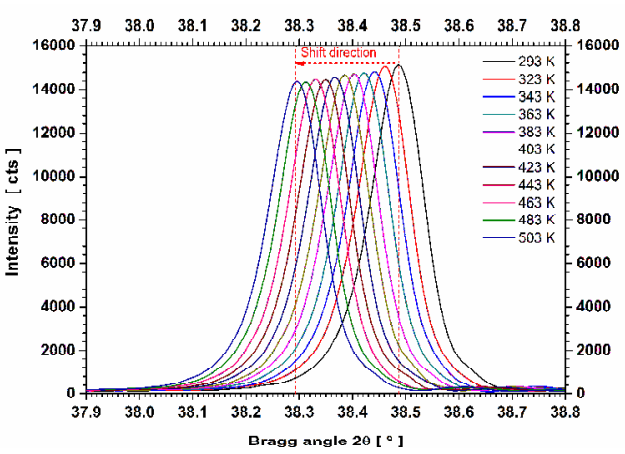

Fig. 2. X-ray profiles comparison of (111)peaks in industrial aluminum samples heated at constant rate

Williamson-Hall method. LPA graphical results are shown in Fig. 3 for industrial aluminum heated from ambient temperature to $503 \mathrm{~K}$ (e.g. at (a) $343 \mathrm{~K}$ and at (b) $443 \mathrm{~K}$ ). Experimental data (blue circles) are reported together with the refined data (red line) and the residual (black line below). The agreement between the experimental and refined patterns, as shown by the residual (the difference between the experimental and refined data), is quite satisfactory. The values obtained for the agreement factors from the LPA refinement are in the range of: $\operatorname{Rwp}=5.25 \sim 6.94 \%$, Rexp $=4.15 \sim 12.81 \%$, and the goodness of fit factor: $\chi^{2}=1.15 \sim 1.76$.
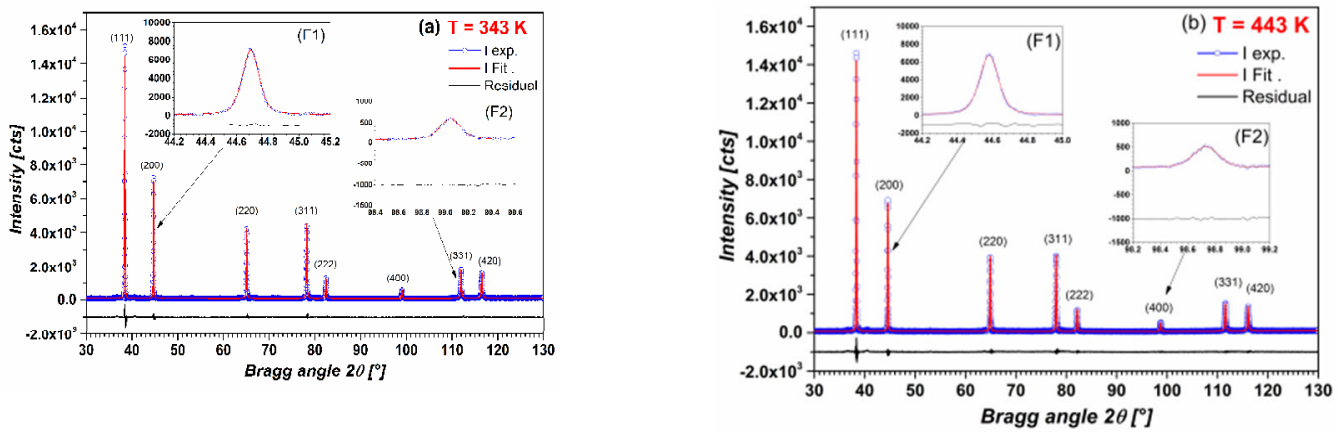

Fig. 3. Graphical ouput of LPA is for industrial Almeasured at (a) $343 \mathrm{~K}$, and (b) $443 \mathrm{~K}$ respectively

Fig. 4 shows the Williamson-Hall plots of industrial aluminum studied at $343 \mathrm{~K}$ and $443 \mathrm{~K}$. The crystallite size and the strain were calculated from the values of the intersection with the ordinate axis and the slope of the fitted line, respectively. 

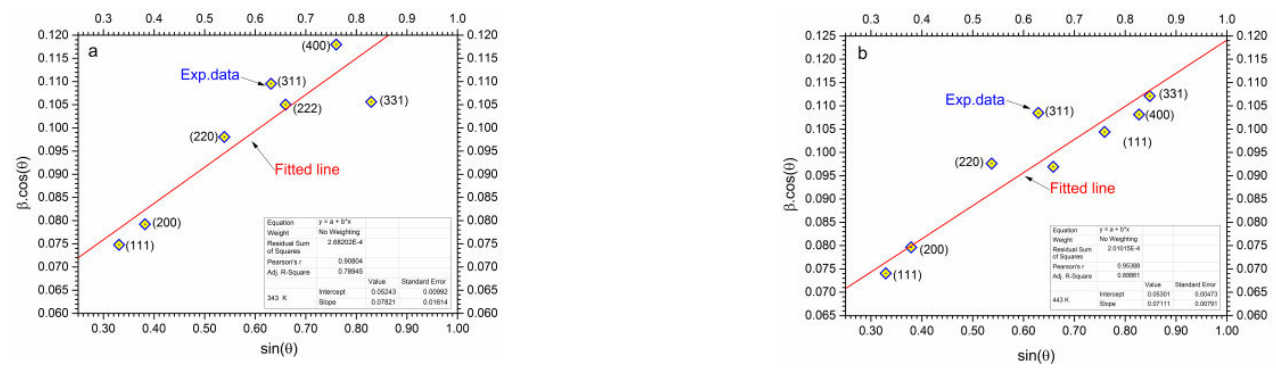

Fig. 4. W-H plots of industrial aluminum correspond to (a) $343 \mathrm{~K}$ and (b) $443 \mathrm{~K}$, temperatures respectively

Table 1 summarizes the LPA refinement results. On one hand, we noted that the size of coherent diffraction domains (crystallites)increases with the temperature rise; on an other hand the strain fluctuates between two limits $36.9 \times 10^{-5}$ and $71.0 \times 10^{-5}$. The external contribution of a quantity of energy in thermal form increases individual kinetic energy of the atoms; the atoms vibrate more and the interatomic distances increase. Therefore, the interplanar spacing grow, there is an effect of expanding the crystal lattice. The lattice parameter and unit cell volume increase $[5,6]$.

Table 1

Summary of microstructural parameters obtained by LPA refinement and by $W$ - $H$ plots

\begin{tabular}{|c|c|r|r|r|r|r|r|r|r|r|}
\hline $\mathrm{T}[\mathrm{K}]$ & \multicolumn{1}{c|}{$\mathbf{2 9 3}$} & $\mathbf{3 2 3}$ & $\mathbf{3 4 3}$ & $\mathbf{3 6 3}$ & \multicolumn{1}{c|}{$\mathbf{3 8 3}$} & $\mathbf{4 2 3}$ & $\mathbf{4 4 3}$ & $\mathbf{4 6 3}$ & $\mathbf{4 8 3}$ & $\mathbf{5 0 3}$ \\
\hline $\mathrm{a}[\mathrm{nm}]$ & 0.40469 & 0.40496 & 0.40515 & 0.40534 & 0.40555 & 0.40590 & 0.40609 & 0.40627 & 0.40646 & 0.40664 \\
\hline dhk1 $[\mathrm{nm}]$ & 0.23375 & 0.23390 & 0.23401 & 0.23412 & 0.23424 & 0.23445 & 0.23455 & 0.23466 & 0.23477 & 0.23487 \\
\hline $\mathrm{D}[\mathrm{nm}]$ & 547 & 609 & 815 & 823 & 943 & 1371 & 2671 & 3128 & 3215 & 3385 \\
\hline$\varepsilon^{\times} \times 10^{-5}$ & 71.0 & 45.1 & 63.9 & 36.9 & 68.2 & 63.3 & 63.8 & 56.3 & 64.9 & 58.8 \\
\hline
\end{tabular}

Conclusion. This study represents a contribution to the investigation of thermal effect on the microstructure properties of industrial aluminum for the manufacture of electric cables. For the X-ray analysis of the profile of the line, we have chosen the so-called «breadth method» whose basic principle is to draw the line versus (Williamson-Hall plot). We noticed the effect of the temperature rise by increasing the crystallite size. XRD detected distortion microstructure involving variation of the lattice parameter and cell parameter, the elevation of temperature leads to an expansion of the unit cell.

\section{REFERENCES}

1. Leoni M., Scardi P. (2000, 09/01/2017) PM2k. Retrieved from http://www.xrd.ing.unitn.it/pm2k/

2. Scherrer P. (1918) Estimation of the size and internal structure of colloidal particles by means of roentgen. Nachrichten von der Gesellschaft der Wissenschaften zu Göttingen, 26(3), pp. 98-100.

3. Khorsand Z.A., Majid W.H., Abrishami M. E., Yousefi R. (2011) X-ray analysis of ZnO nanoparticles by Williamson-Hall and size-strain plot methods. Solid State Sciences, 13(1), pp. 251-256.

4. Williamson G.K., Hall W.H. (1953) X-ray line broadening from filed aluminium and wolfram. Acta Metallurgica, 1, pp. 22-31. doi: http://dx.doi.org/10.1016/0001-6160(53)90006-6

5. Hidnert, P. (1925) Thermal expansion of aluminum and various important aluminum alloys. Journal of the Franklin Institute, 199(4), pp. 539-541.

6. Wilson A.J.C. (1941) The thermal expansion of aluminium from $0^{\circ}$ to $650^{\circ}$ C. Proceedings of the Physical Society, 53(3), pp. 235. 